\title{
Patient-specific prediction model for clinical and quality-of-life outcomes after lumbar spine surgery
}

\author{
${ }^{*}$ Daniel Lubelski, MD, ${ }^{1}$ James Feghali, MD, ${ }^{1}$ Amy S. Nowacki, PhD, $, 3,3$ Vincent J. Alentado, MD, ${ }^{4}$ \\ Ryan Planchard, MD, ${ }^{1}$ Kalil G. Abdullah, MD, ${ }^{5}$ Daniel M. Sciubba, MD, ${ }^{1}$ Michael P. Steinmetz, MD, ${ }^{2,6}$ \\ Edward C. Benzel, MD, ${ }^{2,6}$ and Thomas E. Mroz, MD2,6
}

\begin{abstract}
1Department of Neurosurgery, Johns Hopkins Hospital, Baltimore, Maryland; ${ }^{2}$ Cleveland Clinic Lerner College of Medicine, Cleveland; ${ }^{3}$ Department of Quantitative Health Science, Cleveland Clinic, Cleveland, Ohio; ${ }^{4}$ Department of Neurosurgery, Indiana University School of Medicine, Indianapolis, Indiana; ${ }^{5}$ Department of Neurological Surgery, University of Texas Southwestern Medical Center, Dallas, Texas; and ${ }^{6}$ Department of Neurosurgery and the Cleveland Clinic Center for Spine Health, Cleveland Clinic, Cleveland, Ohio
\end{abstract}

\begin{abstract}
OBJECTIVE Patient demographics, comorbidities, and baseline quality of life (QOL) are major contributors to postoperative outcomes. The frequency and cost of lumbar spine surgery has been increasing, with controversy revolving around optimal management strategies and outcome predictors. The goal of this study was to generate predictive nomograms and a clinical calculator for postoperative clinical and QOL outcomes following lumbar spine surgery for degenerative disease.
\end{abstract}

METHODS Patients undergoing lumbar spine surgery for degenerative disease at a single tertiary care institution between June 2009 and December 2012 were retrospectively reviewed. Nomograms and an online calculator were modeled based on patient demographics, comorbidities, presenting symptoms and duration of symptoms, indication for surgery, type and levels of surgery, and baseline preoperative QOL scores. Outcomes included postoperative emergency department (ED) visit or readmission within 30 days, reoperation within 90 days, and 1-year changes in the EuroQOL-5D (EQ-5D) score. Bootstrapping was used for internal validation.

RESULTS A total of 2996 lumbar surgeries were identified. Thirty-day ED visits were seen in 7\%, 30-day readmission in 12\%, 90-day reoperation in 3\%, and improvement in EQ-5D at 1 year that exceeded the minimum clinically important difference in $56 \%$. Concordance indices for the models predicting ED visits, readmission, reoperation, and dichotomous 1-year improvement in EQ-5D were $0.63,0.66,0.73$, and 0.84 , respectively. Important predictors of clinical outcomes included age, body mass index, Charlson Comorbidity Index, indication for surgery, preoperative duration of symptoms, and the type (and number of levels) of surgery. A web-based calculator was created, which can be accessed here: https://riskcalc.org/PatientsEligibleForLumbarSpineSurgery/.

CONCLUSIONS The prediction tools derived from this study constitute important adjuncts to clinical decision-making that can offer patients undergoing lumbar spine surgery realistic and personalized expectations of postoperative outcome. They may also aid physicians in surgical planning, referrals, and counseling to ultimately lead to improved patient experience and outcomes.

https://thejns.org/doi/abs/10.3171/2020.8.SPINE20577

KEYWORDS prediction; calculator; nomogram; spine surgery; outcome; quality of life; lumbar

$\mathrm{T}$ HE optimal management of many degenerative spinal pathologies remains controversial. ${ }^{1-3}$ Low-back pain resulted in 83 million disability-adjusted life years lost in 2010, ranking highest of the 291 conditions studied in terms of years lived with disability and sixth in overall burden. ${ }^{4,5}$ Elucidating procedure- and patientspecific factors associated with outcomes will be crucial to prospectively identify the subset of patients who would benefit from surgical intervention and to maximize value and outcome for patients. ${ }^{6}$

ABBREVIATIONS ALIF = anterior lumbar interbody fusion; $\mathrm{AUC}=$ area under the curve; $\mathrm{BMI}=$ body mass index; $\mathrm{CCI}=\mathrm{Charlson} \mathrm{Comorbidity} \mathrm{Index;} \mathrm{c}$ index = concordance index; ED = emergency department; EQ-5D = EuroQOL-5D; MCID = minimum clinically important difference; NSQIP = National Surgical Quality Improvement Program; PDQ = Pain and Disability Questionnaire; PLIF = posterior lumbar interbody fusion; $\mathrm{QOL}=$ quality of life; TLIF = transforaminal lumbar interbody fusion. SUBMITTED April 13, 2020. ACCEPTED August 11, 2020.

INCLUDE WHEN CITING Published online January 29, 2021; DOI: 10.3171/2020.8.SPINE20577.

${ }^{*}$ D.L. and J.F. contributed equally to this work. 
Evidence evaluating lumbar spine surgery for degenerative disease remains inconsistent for many indications. ${ }^{7}$ Particularly for degenerative lumbar spinal disease, there is mixed evidence regarding which treatments are best in improving outcomes. The Spine Patient Outcomes Research Trial (SPORT) questioned the superiority of surgery versus nonoperative treatment. ${ }^{8}$ Recent randomized trials in the United States by Ghogawala et al. ${ }^{9}$ and in Sweden by Försth and colleagues ${ }^{10}$ had conflicting results as to the success of decompression versus fusion for patients with lumbar spinal stenosis and spondylolisthesis. Substantial variability exists regarding optimal treatments for various interventions for lumbar spinal pathology. ${ }^{1-3}$ As we continue to refine the understanding of these pathologies and their optimal treatment, patient/procedural factors associated with outcomes are increasingly important to elucidate.

There have been a multitude of studies describing associations between preoperative factors and postoperative complications, adverse outcomes, and quality-of-life (QOL) outcomes following lumbar spine surgery. ${ }^{11-13}$ However, these associations are difficult to interpret for a given patient with a unique set of baseline characteristics that may confer both a negative and a positive prognosis of success of a given treatment. Nomograms have emerged as a useful, readily interpretable, and intuitive way of using evidence-based models to understand and construct outcome predictions..$^{14}$ They also offer a visual representation of the computational framework underlying predictive models that a patient can easily grasp..$^{14}$ Similar to previous models predicting outcomes after cervical spine surgery, ${ }_{15}^{15}$ we sought to develop nomograms and a webbased calculator tool that could use patient-, symptom-, and surgery-specific variables to predict outcomes following lumbar spine surgery for degenerative disease.

\section{Methods}

A prospectively maintained database of consecutive patients who underwent anterior or posterior lumbar decompression and/or fusion between June 2009 and December 2012 was retrospectively queried for this study after obtaining institutional review board approval. Exclusion criteria included patients younger than 18 years of age and patients with surgery indications other than degenerative disease (such as infection, tumor, trauma). The database contained data on patient demographics, comorbidities, previous medical/surgical history, presenting symptoms, and procedural details. The selection of candidate predictors for evaluation in this study was motivated by an extensive review of the relevant literature. Outcome measures included all-cause 30-day emergency department (ED) visits/readmissions, reoperation within 90 days for infection, and the EuroQOL-5D (EQ-5D) outcome. QOL was selected given its direct relevance to the goals of surgery. It also captures elements of other outcome measures, such as need for rehabilitation and complications (predictors of QOL). ED visits, readmission, and reoperation constitute other variables that are relevant to value-centric care and are of interest to patients, surgeons, and hospital systems.

\section{Health-Related QOL}

QOL metrics (EQ-5D) are prospectively collected via the institutional Knowledge Program. The Knowledge Program is a tool integrated within the institutional electronic medical records that collects and organizes patientspecific health-related QOL metrics at every outpatient visit. Preoperative QOL scores were collected within 1 month prior to the date of surgery, and the postoperative QOL scores used for analysis were obtained closest to 1 year after the date of surgery. The minimum clinically important difference (MCID) defined for EQ-5D in a 1-year period was 0.1 quality-adjusted life year. ${ }^{16,17}$

\section{Statistical Analysis}

Analysis was conducted using $\mathrm{R}$ statistical software ( $\mathrm{R}$ Foundation for Statistical Computing). In describing the study sample, categorical data were summarized with counts (percent), and continuous variables were summarized using the mean and standard deviation. Depending on the specific outcome, the sample size available for the model ranged from 273 to 2481 subjects, because listwise deletion rather than imputation was used in the case of missing values. Multivariable logistic regression was used to create prediction models for the binary outcomes (postoperative ED visit within 30 days, readmission within 30 days, reoperation within 90 days, 1-year improvement in each QOL score) using variables with a $\mathrm{p}$ value $<0.3$ on univariable analysis. Linearity assumptions of the numeric predictor variables were relaxed using restricted cubic splines. ${ }^{18}$

For the binary outcomes, each final model was identified as that which achieved the maximum bias-corrected concordance index (c index) (ClevClinicQHS R package). The $\mathrm{c}$ index characterizes the discriminatory capability of a model and is defined as the probability that given two randomly selected patients, the patient with the worse outcome was, in fact, predicted to have a worse outcome. This measure, similar to an area under the curve (AUC), ranges from 0.5 (chance or a coin flip) to 1.0 (perfect ability to rank patients). To correct for the optimism bias inherent in assessing model performance on the data used to generate the model, a bootstrap validation was performed. One thousand bootstrap samples (sampling with replacement) were drawn and the final model was fit to each sample. Predicted probabilities were obtained for the original sample based on each bootstrap estimated model and a c index was calculated resulting in 1000 bootstrap c indices. The bias-corrected $\mathrm{c}$ index is defined as the average of these bootstrap c indices. Model calibration was measured by plotting the proportion of patients predicted to develop each outcome against the actual proportion who developed each outcome for the original sample and the bootstrap samples. Values that closely follow the $45^{\circ}$ line of equality (ideal line) signify high model calibration. Finally, the Rshiny package was used to design web-based calculators created from the models.

\section{Results \\ Descriptive Data}

The study sample consisted of 2996 patients undergoing surgery for lumbar degenerative spine disease. Patient- 
TABLE 1. Patient-related and operative characteristics in 2996 patients who underwent lumbar spine surgery for degenerative disease

\begin{tabular}{|c|c|c|}
\hline Characteristic & Value & No. of Pts \\
\hline \multicolumn{3}{|l|}{ Demographics } \\
\hline Age, yrs & $58.3 \pm 15.0$ & 2996 \\
\hline Male & $1610(54)$ & 2996 \\
\hline White race & $2632(88)$ & 2996 \\
\hline Married & $2082(69)$ & 2996 \\
\hline $\mathrm{BMI}, \mathrm{kg} / \mathrm{m}^{2}$ & $29.5 \pm 5.8$ & 1981 \\
\hline Current or former smoker & $214(16)$ & 1379 \\
\hline Median income & $\$ 54,800 \pm \$ 17,100$ & 2019 \\
\hline $\mathrm{CCl}$ & $2.8 \pm 1.5$ & 2202 \\
\hline Baseline PDQ score & $85 \pm 29$ & 1503 \\
\hline Symptom duration, mos & $24.1 \pm 39.7$ & 2958 \\
\hline Clinical symptoms & & 2996 \\
\hline Low-back pain & $2176(73)$ & \\
\hline Radiculopathy & $542(18)$ & \\
\hline Lower-extremity weakness & $244(8)$ & \\
\hline Bowel/bladder symptoms & $8(0.3)$ & \\
\hline Indications for surgery & & 2996 \\
\hline Foraminal stenosis & $487(16)$ & \\
\hline Spinal stenosis & $1580(53)$ & \\
\hline Herniation & $950(32)$ & \\
\hline Spondylolisthesis & $773(26)$ & \\
\hline Radiculopathy & $542(18)$ & \\
\hline Previous surgery & $280(9)$ & 2969 \\
\hline Type of surgery & & 2996 \\
\hline ALIF & $228(8)$ & \\
\hline PLIF/TLIF & $481(16)$ & \\
\hline Decompression & $1655(55)$ & \\
\hline Posterolateral fusion & $930(31)$ & \\
\hline Levels & $1.7 \pm 1.4$ & 2972 \\
\hline
\end{tabular}

Pts $=$ patients.

Values are expressed as either the number (\%) or the mean \pm SD

related and operative characteristics are summarized in Table 1. Patients had a mean age of 58.3 years, and $54 \%$ were male. The mean body mass index (BMI) was $29.5 \mathrm{~kg} /$ $\mathrm{m}^{2}$, and the Charlson Comorbidity Index (CCI) was 2.8. Most patients were White (88\%) and married (69\%), and
$16 \%$ were current or former smokers. Low-back pain was the most frequently reported presenting clinical symptom (73\%), followed by radiculopathy (18\%), lower-extremity weakness $(8 \%)$, and bowel/bladder symptoms $(0.3 \%)$. Decompression was the most common type of surgery performed (55\%), with spinal stenosis being the most common indication (53\%), and $9 \%$ of patients had undergone previous surgery. The mean number of operated levels was 1.7.

Within 30 days after surgery, $7 \%$ of patients visited the $\mathrm{ED}$, and $12 \%$ of patients were readmitted. In the 90-day postoperative period, a reoperation procedure was required in 3\% of patients (Table 2). Concerning the EQ-5D, the mean preoperative, 1-year postoperative, and 1-year change scores were $0.50,0.68$, and +0.18 quality-adjusted life years, respectively (Table 2), with $56 \%$ of patients achieving improvement greater than the MCID.

\section{Outcome Predictors}

The model predictive of 30-day visits to the ED consisted of the following predictors: race, marital status, symptom duration, foraminal stenosis, disc herniation, spondylolisthesis, radiculopathy, anterior lumbar interbody fusion (ALIF), posterior lumbar interbody fusion (PLIF) or transforaminal lumbar interbody fusion (TLIF), posterolateral lumbar fusion, decompression, and number of operated levels (Fig. 1; Supplemental Table 1). The c index was 0.63 .

The model predictive of 30-day readmission consisted of the following predictors: BMI, CCI, disc herniation, decompression, and number of operated levels (Fig. 2; Supplemental Table 2). The model provides modest discrimination with an apparent $\mathrm{c}$ index of 0.66 .

The model predictive of 90-day reoperation related to infection consisted of the following predictors: age, BMI, CCI, spinal stenosis, ALIF, posterolateral lumbar fusion, and number of operated levels (Fig. 3; Supplemental Table $3)$. The model provides good discrimination with an apparent c index of 0.73 .

Predictors included in the final model predictive of favorable 1-year postoperative EQ-5D outcome were as follows: race, foraminal stenosis, disc herniation, spondylolisthesis, radiculopathy, preoperative EQ-5D score, and preoperative Pain and Disability Questionnaire (PDQ) score (Fig. 4; Supplemental Table 4). The model's apparent $\mathrm{c}$ index was 0.84 .

A risk calculator (Fig. 5) was available under the following URL: https://riskcalc.org/PatientsEligibleFor LumbarSpineSurgery/.

TABLE 2. Outcomes in 2996 patients who underwent lumbar spine surgery for degenerative disease

\begin{tabular}{l}
\hline \multicolumn{2}{c}{ Outcome } & \multicolumn{2}{c}{ Value } \\
\hline 30-day ED visits \\
30-day readmissions
\end{tabular}


Married

Symptom Duration

Foraminal Stenosis

Disc Herniation

Spondylolisthesis

Radiculopathy

ALIF

PLIF / TLIF

Posterolateral

Decompression

Levels

Total Points

Predicted Probability of ED Visit within 30 Days
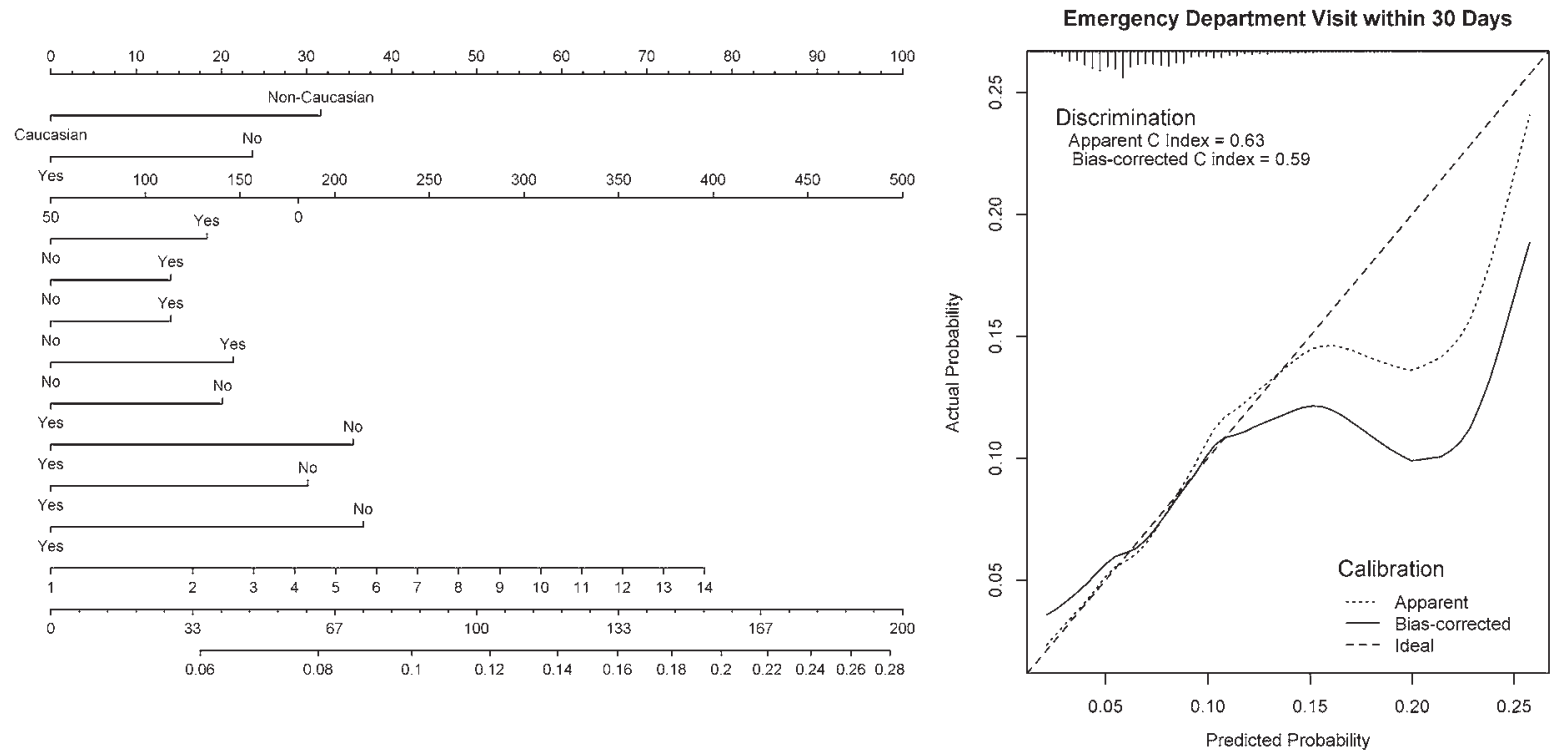

FIG. 1. Nomogram representing prediction model for 30-day visits to the ED with associated prediction performance $(\mathrm{n}=2481)$.

\section{Discussion}

The number of operations and total cost for lumbar degenerative disease have increased in recent decades..$^{19,20}$ Between 2004 and 2015, there was a 62.3\% increase in the volume of elective lumbar fusion, with a concomitant $177 \%$ increase in aggregate hospital costs. ${ }^{21}$ In view of these trends and the increased emphasis on value-centric care, the ability to predict unfavorable outcome can optimize patient selection for surgery. Because patient satisfaction may improve with increased involvement in the decision-making process, ${ }^{22,23}$ there is value in using nomograms and other such prediction tools that can provide patients with realistic and individualized expectations after surgery.

\section{ED Visits}

Our cohort had a 30-day ED visit rate of 7\%, with demographic, disease-related, and procedure-related factors all being important predictors. Although factors associated with postoperative ED visits after lumbar spine surgery have been described previously, no bedside individualized predictive tool has been designed. Predicting the likelihood of an ED visit after lumbar spine surgery should be of interest to hospitals seeking to improve the quality of their care, because postoperative ED visits in this setting are linked to lower Hospital Consumer Assessment of Healthcare Providers and Systems scores. ${ }^{24}$ An analysis by Hills et al. of 2762 patients undergoing elective spine surgery for degenerative disease $(64 \%$

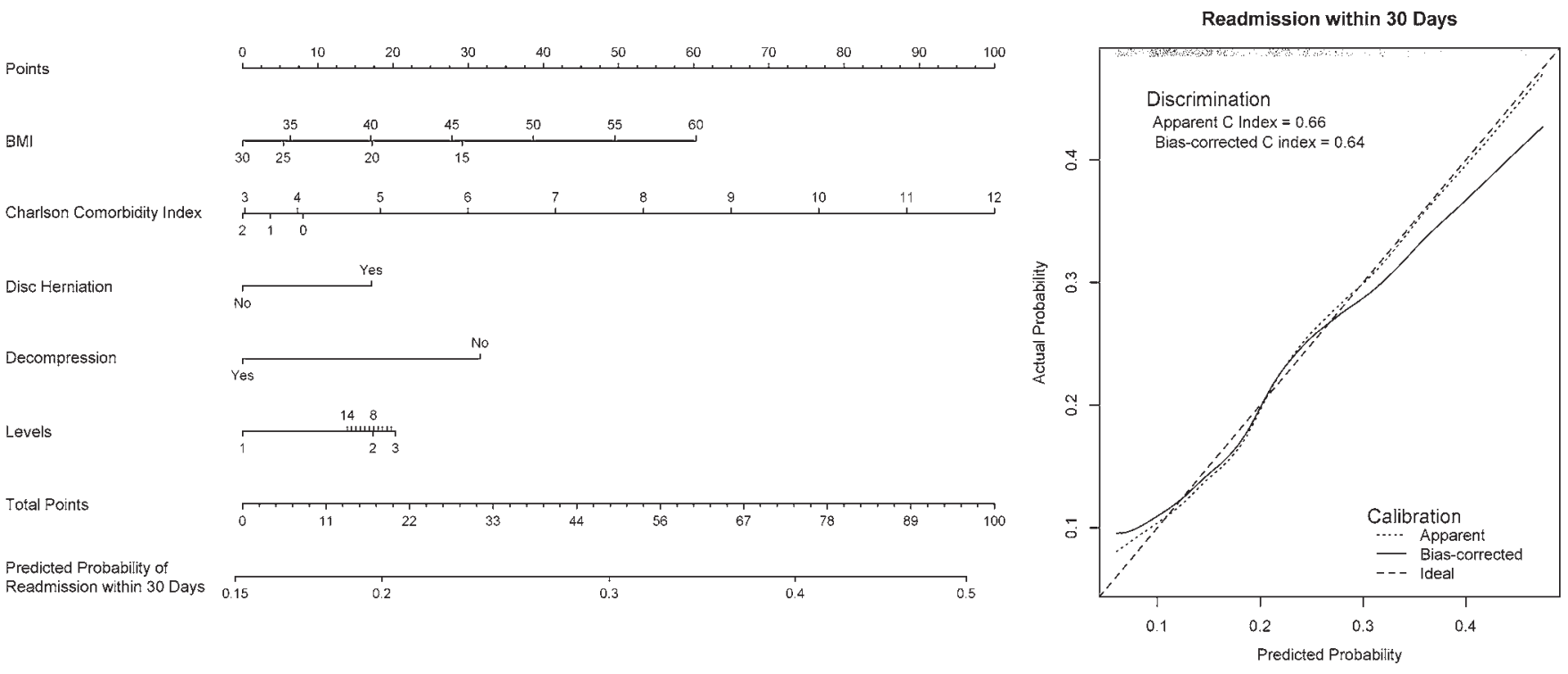

FIG. 2. Nomogram representing prediction model for 30-day readmission with associated prediction performance $(n=1274)$. 

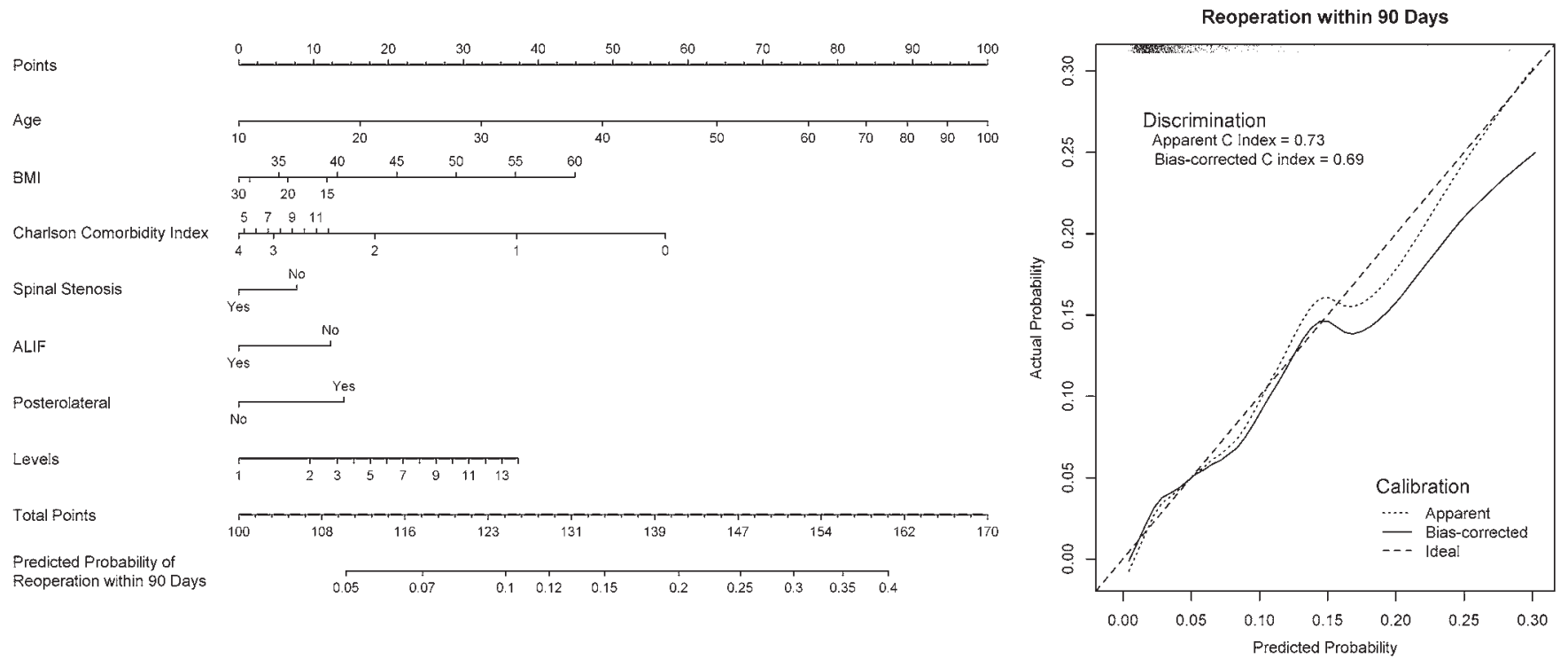

FIG. 3. Nomogram representing prediction model for 90 -day reoperation for infection with associated prediction performance $(n=$ 1489).

lumbar) reported a 90-day ED visit rate of 9.4\%, and independent risk factors were younger age, baseline opioid intake, chronic obstructive pulmonary disorder, and a larger number of surgically treated vertebral levels. ${ }^{25}$ Patients with a 90-day ED visit also had a significantly lower 3-month EQ-5D score. ${ }^{25}$ Another study by Jain et al. focusing on primary lumbar fusion reported a 30-day ED visit rate of $12.8 \%$ with some similar predictors to our model, including Black race, ALIF, and $\geq 3$ surgically treated levels. ${ }^{26}$ ALIF operations have been shown to be associated with increased rates of postoperative complications, readmissions, and hospital costs. ${ }^{27}$ Additionally, longer-level fusions ( $\geq 3$ levels) have been associated with a greater incidence of postsurgical complications. ${ }^{28}$ These findings may account for the higher ED visit rate among patients with these risk factors.

\section{Readmissions}

The 30-day readmission rate in this study was $12 \%$ and was best predicted by higher BMI, higher CCI, disc herniation, and an increased number of surgically treated levels. A study on all lumbar spine surgeries (any indication) queried from the National Surgical Quality Improvement Program (NSQIP) database reported a 30-day readmission rate of $4.4 \%$, with the most common cause being wound-related complications (38.6\%). ${ }^{29}$ The authors reported an increase in readmission rates with increased procedure invasiveness

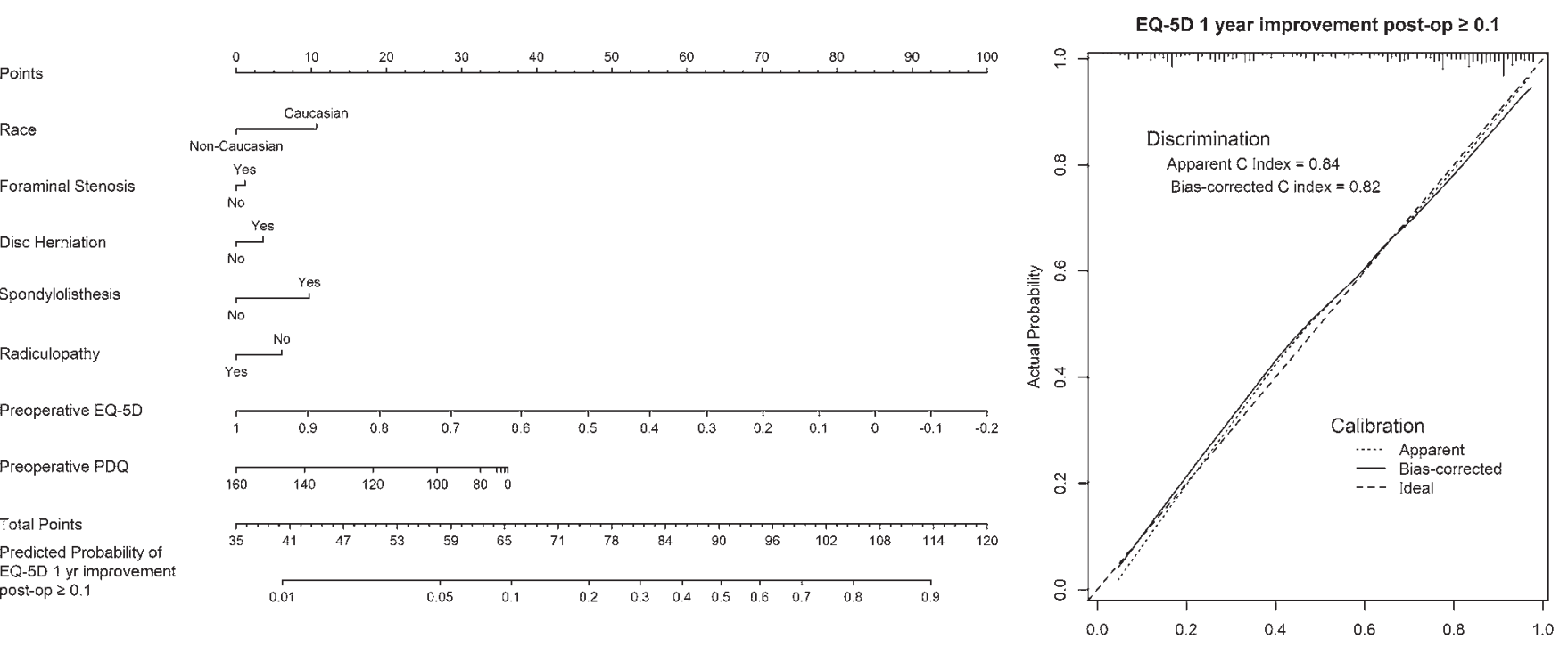

FIG. 4. Nomogram representing prediction model for 1-year dichotomized improvement in EQ-5D score with associated prediction performance $(n=418)$. 
A

Age in years

58

Race

Caucasian

Married

Yes

Body Mass Index (kg/m^2)

25

Charlson Comorbidity Index

3

Symptom Duration in months

6

Foraminal Stenosis

No

Spinal Stenosis

Yes

Discherniation

No

B

Age in years
78
Race
Caucasian
Married
Yes

Body Mass Index (kg/m^2)

35

Charlson Comorbidity Index

5

Symptom Duration in months

Foraminal Stenosis

Yes

Spinal Stenosis

Yes

Disc herniation

No
Spondylolisthesis

No

Run Calculator

Radiculopathy

Yes

\begin{tabular}{llll} 
& & Result & Probability \\
\cline { 2 - 4 } ALIF & 1 & Predicted Probability of Emergency Department Visit within 30 Days & $5.9 \%$
\end{tabular}

$\begin{array}{llll}2 & \text { Predicted Probability of Readmission within } 30 \text { Days } & 7 \%\end{array}$

No $3 \quad$ Predicted Probability of Reoperation for Infection within 90 Days $\quad 0.7 \%$

Posterolateral Fusion $\quad 4 \quad$ Predicted Probability of 1 Year Improvement in EQ-5D Greater than $0.1 \quad 75.7 \%$

No

Decompression

Yes

Number of Levels

1

Preoperative EQ-5D (optional)

0.4

Preoperative PDQ (optional)

20

Spondylolisthesis

Run Calculator

Yes

Radiculopathy

Yes

1 Predicted Probability of Emergency Department Visit within 30 Days $5.1 \%$

2 Predicted Probability of Readmission within 30 Days $18.8 \%$

No 3 Predicted Probability of Reoperation for Infection within 90 Days $9.2 \%$

PLIF/TUF 4 Predicted Probability of 1 Year Improvement in EQ-5D Greater than $0.141 .1 \%$

Yes

Posterolateral Fusion

Yes

Decompression

Yes

Number of Levels

3

Preoperative EQ-5D (optional)

0.6

Preoperative PDQ (optional)

100

FIG. 5. Screenshot of the web-based calculator. A: Panel showing prediction in a 58-year-old patient with a BMI of 25 undergoing a single-level lumbar decompression for radiculopathy with a low predicted probability of ED visit, readmission, or reoperation for infection and a high probability of EQ-5D improvement. B: In contrast, this panel shows the predicted outcomes of a 78-yearold patient with a BMI of 35 and extensive comorbidities who undergoes 3-level decompression and posterolateral fusion with PLIF for stenosis and spondylolisthesis; here one can see the predicted outcome of a greater chance of infection, readmission, and ED visit within 30 days. Use of this type of calculator could facilitate preoperative discussion, weighing of risk and benefit, and better understanding of expected hospital resource use. The calculator we used can be accessed at https://riskcalc.org/ PatientsEligibleForLumbarSpineSurgeryl. Figure is available in color online only. 
and comorbidity burden, ${ }^{29}$ similar to the predictors identified in our analysis. A more focused query of elective laminectomy procedures for lumbar spinal stenosis in NSQIP identified age, BMI, comorbidity burden, and steroid use as independent risk factors for readmission, which also corroborates the findings of our study. ${ }^{30} \mathrm{In}$ an analysis of 1803 consecutive patients undergoing lumbar spine surgery for degenerative disease, the readmission rate was $6 \%$, and a predictive model based on more than 40 variables yielded an AUC of $0.74 .{ }^{31}$ In our model, decompression-only procedures were associated with a lower risk of readmission compared to other procedures. This is in line with the findings of Akamnonu et al., who reported almost double the readmission rate in fusion procedures $(3.9 \%)$ compared to decompression procedures $(2.1 \%)$ in the surgical treatment of common lumbar pathologies. ${ }^{32}$ A previous model published by Wang et al. consisted of the following predictors of 30-day readmission after elective lumbar spine surgery among Medicare beneficiaries: age, Black race, comorbidity burden, Medicare/Medicaid eligibility, number of surgically treated levels, fusion surgery, and anterior surgical approach; the AUC in their study was 0.61 compared to the slightly higher 0.66 in the current study. ${ }^{33}$ Similarly, McGirt and colleagues reported the Carolina-Semmes Grading Scale, a model predicting 90-day hospital readmissions following elective lumbar spine surgery, which identified factors such as age and comorbidity burden, and also had a slightly lower AUC of $0.58 .^{34}$

\section{Reoperations}

The 90-day reoperation rate for infection was $3 \%$ and was best predicted by older age, higher BMI, higher CCI, posterolateral lumbar fusion, and an increasing number of surgically treated levels. An NSQIP-based analysis of patients undergoing PLIF reported a comparable reoperation rate of $3.7 \%$ over 30 days, and similarly identified increased comorbidity (American Society of Anesthesiologists class $>$ II) as a predictor of reoperation. ${ }^{35}$ The inclusion of BMI as a significant predictor confirms previous findings such as the independent association between obesity and reoperation after lumbar laminectomy, ${ }^{36}$ and minimally invasive lumbar discectomy. ${ }^{37}$ Another reason for the association between increased BMI and reoperation in lumbar degenerative disease is the increased incidence of complications such as surgical site infections following initial lumbar surgery. ${ }^{38}$ Our model showed that patients undergoing posterolateral fusions had a higher likelihood of undergoing reoperation. Martin et al. evaluated reoperation rates following lumbar spine surgery in patients with degenerative disease and reported a significantly higher incidence of reoperation over an 11-year period in patients with diseases other than spondylolisthesis who underwent fusion compared with decompression only (adjusted HR 1.41). ${ }^{39}$ Reoperations were more commonly linked to either device complications or pseudarthrosis and not disease progression. ${ }^{39}$

\section{Health-Related QOL}

Concerning QOL outcomes, 75\% of patients demonstrated improvement in preoperative to postoperative QOL and $56 \%$ achieved a clinically relevant (MCID) 1-year improvement in EQ-5D scores. Key predictors of significant improvement included White race, lower baseline EQ-5D and PDQ scores, and indications such as spondylolisthesis, disc herniation, and radiculopathy. The predictive power achieved for this outcome was one of the highest reported in the literature (apparent $\mathrm{c}$ index $=0.84$ ). Several studies described improvements in various QOL measures following lumbar spine surgery. A study on documented operations for lumbar spinal stenosis in the National Swedish Registry similarly reported an improvement in the mean EQ-5D score from 0.36 to 0.64 at 1 year after surgery. ${ }^{40}$ Based on an analysis of the Quality Outcomes Database, McGirt et al. reported significant improvement in several patient-reported outcomes 1 year after lumbar spine surgery. ${ }^{41}$ Namely, the EQ-5D improved from a median preoperative score of 0.6 to a median postoperative score of 0.82 at 1 year after surgery. ${ }^{41}$ Their predictive model for EQ-5D had modest predictive performance $(\mathrm{AUC}=0.69)$ and was based on 17 different variables including age, preoperative QOL, baseline disability score, comorbidity burden, obesity, and race. ${ }^{41}$ BMI was not a significant predictor in our study, consistent with the findings of Sielatycki et al., who reported no significant association between a high BMI $\left(\geq 35 \mathrm{~kg} / \mathrm{m}^{2}\right)$ and 1-year EQ-5D change after lumbar surgery for degenerative disease. ${ }^{42}$

\section{Limitations}

The findings of this study must be interpreted with caution. Although data on EQ-5D were entered prospectively, many of the other clinical variables were collected through a retrospective review of the electronic medical records. In addition, some variables had missing data values, which limited the sample size available to derive the final models. However, we do not expect a high degree of bias because the likelihood of missing data is not related to baseline variables or outcome. Given that the data were derived from a tertiary referral center, we expect our findings to be more likely to be generalizable to similar highvolume institutions that take care of a large proportion of complex spinal pathology. Ultimately, multicenter external validation of these prediction models will be needed before widespread use. The incorporation of other characteristics such as narcotic use/abuse, psychiatric comorbidity, and insurance coverage may also improve prediction accuracy and generalizability. Finally, QOL, ED visits/readmissions, and reoperations are only a few of the important outcome measures that are relevant to lumbar spine surgery. Length of stay, discharge disposition, and symptom improvement all constitute additional outcome measures that should be considered in the shared decision-making process.

\section{Conclusions}

This study looked at a large database of patients who underwent lumbar spine surgery for degenerative disease and yielded an intuitive prediction calculator and nomograms for clinically relevant outcomes including 30-day ED visits, 30-day readmissions, 90-day reoperations, and 1-year EQ-5D score improvement. These tools can serve as useful adjuncts to clinical decision-making and as an intuitive communication medium when advising patients in the context of informed consent. 


\section{Acknowledgments}

We thank the Neurological Institute Knowledge Project.

\section{References}

1. Mroz TE, Lubelski D, Williams SK, et al. Differences in the surgical treatment of recurrent lumbar disc herniation among spine surgeons in the United States. Spine J. 2014;14(10): 2334-2343.

2. Lubelski D, Williams SK, O'Rourke C, et al. Differences in the surgical treatment of lower back pain among spine surgeons in the United States. Spine (Phila Pa 1976). 2016; 41(11):978-986.

3. Lubelski D, Alentado VJ, Williams SK, et al. Variability in surgical treatment of spondylolisthesis among spine surgeons. World Neurosurg. 2018;111:e564-e572.

4. Ravindra VM, Senglaub SS, Rattani A, et al. Degenerative lumbar spine disease: estimating global incidence and worldwide volume. Global Spine J. 2018;8(8):784-794.

5. Hoy DG, March LM, Brooks P, et al. The global burden of low back pain: estimates from the Global Burden of Disease 2010 study. Ann Rheum Dis. 2014;73:968-974.

6. Parker SL, Chotai S, Devin CJ, et al. Bending the cost curve-establishing value in spine surgery. Neurosurgery. 2017;80(3S):S61-S69.

7. Mirza SK, Deyo RA. Systematic review of randomized trials comparing lumbar fusion surgery to nonoperative care for treatment of chronic back pain. Spine (Phila Pa 1976). 2007; 32(7):816-823.

8. Lurie JD, Tosteson TD, Tosteson A, et al. Long-term outcomes of lumbar spinal stenosis: eight-year results of the Spine Patient Outcomes Research Trial (SPORT). Spine (Phila Pa 1976). 2015;40(2):63-76.

9. Ghogawala Z, Dziura J, Butler WE, et al. Laminectomy plus fusion versus laminectomy alone for lumbar spondylolisthesis. N Engl J Med. 2016;374(15):1424-1434.

10. Försth P, Ólafsson G, Carlsson T, et al. A randomized, controlled trial of fusion surgery for lumbar spinal stenosis. $N$ Engl J Med. 2016;374(15):1413-1423.

11. Ulrich NH, Burgstaller JM, Gravestock I, et al. Outcome of unilateral versus standard open midline approach for bilateral decompression in lumbar spinal stenosis: is "over the top" really better? A Swiss prospective multicenter cohort study. $J$ Neurosurg Spine. 2019;31(2):236-245.

12. Khan I, Bydon M, Archer KR, et al. Impact of occupational characteristics on return to work for employed patients after elective lumbar spine surgery. Spine J. 2019;19(12):19691976.

13. Zakaria HM, Mansour TR, Telemi E, et al. Use of Patient Health Questionnaire-2 scoring to predict patient satisfaction and return to work up to 1 year after lumbar fusion: a 2-year analysis from the Michigan Spine Surgery Improvement Collaborative. J Neurosurg Spine. 2019;31(6):794-801.

14. Kattan MW, Marasco J. What is a real nomogram? Semin Oncol. 2010;37(1):23-26.

15. Lubelski D, Alentado V, Nowacki AS, et al. Preoperative nomograms predict patient-specific cervical spine surgery clinical and quality of life outcomes. Neurosurgery. 2018; 83(1):104-113.

16. Norman GR, Sloan JA, Wyrwich KW. Interpretation of changes in health-related quality of life: the remarkable universality of half a standard deviation. Med Care. 2003;41(5): 582-592.

17. Parker SL, Godil SS, Shau DN, et al. Assessment of the minimum clinically important difference in pain, disability, and quality of life after anterior cervical discectomy and fusion: clinical article. J Neurosurg Spine. 2013;18(2):154-160.

18. Harrell FE Jr, Lee KL, Mark DB. Multivariable prognostic models: issues in developing models, evaluating assumptions and adequacy, and measuring and reducing errors. Stat Med. 1996;15(4):361-387.

19. Yoshihara H, Yoneoka D. National trends in the surgical treatment for lumbar degenerative disc disease: United States, 2000 to 2009. Spine J. 2015;15(2):265-271.

20. Pannell WC, Savin DD, Scott TP, et al. Trends in the surgical treatment of lumbar spine disease in the United States. Spine J. 2015;15(8):1719-1727.

21. Martin BI, Mirza SK, Spina N, et al. Trends in lumbar fusion procedure rates and associated hospital costs for degenerative spinal diseases in the United States, 2004 to 2015. Spine (Phila Pa 1976). 2019;44(5):369-376.

22. Clark JA, Wray NP, Ashton CM. Living with treatment decisions: regrets and quality of life among men treated for metastatic prostate cancer. J Clin Oncol. 2001;19(1):72-80.

23. Deyo RA, Cherkin DC, Weinstein J, et al. Involving patients in clinical decisions: impact of an interactive video program on use of back surgery. Med Care. 2000;38(9):959-969.

24. Levin JM, Winkelman RD, Smith GA, et al. Emergency department visits after lumbar spine surgery are associated with lower Hospital Consumer Assessment of Healthcare Providers and Systems scores. Spine J. 2018;18(2):226-233.

25. Hills JM, Khan I, Sivaganesan A, et al. Emergency department visits after elective spine surgery. Neurosurgery. 2019; 85(2):E258-E265.

26. Jain N, Brock JL, Phillips FM, et al. 30-day emergency department visits after primary lumbar fusion: incidence, causes, risk factors, and costs. Clin Spine Surg. 2019;32(3):113-119.

27. Qureshi R, Puvanesarajah V, Jain A, et al. A comparison of anterior and posterior lumbar interbody fusions: complications, readmissions, discharge dispositions, and costs. Spine (Phila Pa 1976). 2017;42(24):1865-1870.

28. Lee JK, Kim CW, Kang C-N. Long-term outcomes of long level posterolateral fusion in lumbar degenerative disease: comparison of long level fusion versus short level fusion: a case control study. BMC Musculoskelet Disord. 2015;16:381.

29. Pugely AJ, Martin CT, Gao Y, Mendoza-Lattes S. Causes and risk factors for 30-day unplanned readmissions after lumbar spine surgery. Spine (Phila Pa 1976). 2014;39(9):761-768.

30. Basques BA, Varthi AG, Golinvaux NS, et al. Patient characteristics associated with increased postoperative length of stay and readmission after elective laminectomy for lumbar spinal stenosis. Spine (Phila Pa 1976). 2014;39(10):833-840.

31. McGirt MJ, Sivaganesan A, Asher AL, Devin CJ. Prediction model for outcome after low-back surgery: individualized likelihood of complication, hospital readmission, return to work, and 12-month improvement in functional disability. Neurosurg Focus. 2015;39(6):E13.

32. Akamnonu C, Cheriyan T, Goldstein JA, et al. Unplanned hospital readmission after surgical treatment of common lumbar pathologies: rates and causes. Spine (Phila Pa 1976). 2015;40(6):423-428.

33. Wang MC, Shivakoti M, Sparapani RA, et al. Thirty-day readmissions after elective spine surgery for degenerative conditions among US Medicare beneficiaries. Spine J. 2012; 12(10):902-911.

34. McGirt MJ, Parker SL, Chotai S, et al. Predictors of extended length of stay, discharge to inpatient rehab, and hospital readmission following elective lumbar spine surgery: introduction of the Carolina-Semmes Grading Scale. J Neurosurg Spine. 2017;27(4):382-390.

35. Cloney MB, Garcia RM, Smith ZA, Dahdaleh NS. The effect of steroids on complications, readmission, and reoperation after posterior lumbar fusion. World Neurosurg. 2018;110: e526-e 533.

36. Bydon M, Macki M, De la Garza-Ramos R, et al. Smoking as an independent predictor of reoperation after lumbar laminectomy: a study of 500 cases. J Neurosurg Spine. 2015; 22(3):288-293. 
37. Bohl DD, Ahn J, Mayo BC, et al. Does greater body mass index increase the risk for revision procedures following a single-level minimally invasive lumbar discectomy? Spine (Phila Pa 1976). 2016;41(9):816-821.

38. De la Garza-Ramos R, Bydon M, Abt NB, et al. The impact of obesity on short- and long-term outcomes after lumbar fusion. Spine (Phila Pa 1976). 2015;40(1):56-61.

39. Martin BI, Mirza SK, Comstock BA, et al. Reoperation rates following lumbar spine surgery and the influence of spinal fusion procedures. Spine (Phila Pa 1976). 2007;32(3):382-387.

40. Jansson KA, Németh G, Granath F, et al. Health-related quality of life (EQ-5D) before and one year after surgery for lumbar spinal stenosis. J Bone Joint Surg Br. 2009;91(2):210-216.

41. McGirt MJ, Bydon M, Archer KR, et al. An analysis from the Quality Outcomes Database, Part 1. Disability, quality of life, and pain outcomes following lumbar spine surgery: predicting likely individual patient outcomes for shared decisionmaking. J Neurosurg Spine. 2017;27(4):357-369.

42. Sielatycki JA, Chotai S, Stonko D, et al. Is obesity associated with worse patient-reported outcomes following lumbar surgery for degenerative conditions? Eur Spine J. 2016;25(5): $1627-1633$

\section{Disclosures}

Dr. Mroz receives royalties from Stryker. Dr. Sciubba is a consultant for DePuy-Synthes, Medtronic, Stryker, and Baxter. Dr. Steinmetz receives royalties from Zimmer-Biomet and Elsevier, and honoraria from Stryker and Globus.

\section{Author Contributions}

Conception and design: Lubelski. Acquisition of data: Lubelski, Alentado. Analysis and interpretation of data: all authors. Drafting the article: Lubelski, Feghali, Planchard. Critically revising the article: Mroz, Lubelski, Feghali, Nowacki, Abdullah, Sciubba, Steinmetz, Benzel. Reviewed submitted version of manuscript: all authors. Statistical analysis: Feghali, Nowacki.

\section{Supplemental Information}

Online-Only Content

Supplemental material is available with the online version of the article.

Supplemental Tables 1-4. https://thejns.org/doi/suppl/10.3171/ 2020.8.SPINE20577.

\section{Correspondence}

Thomas E. Mroz: Neurological Institute, Cleveland Clinic Center for Spine Health, The Cleveland Clinic, Cleveland, OH. mrozt@ ccf.org. 